\title{
Influence of microstructure evolution during thermal aging on tensile properties of $\beta$-type Ti-12V-2Fe-Al alloy
}

\author{
Weilin Wang, Xianbing Zhang and Jian Sun*
}

Shanghai Key Laboratory of Advanced High-Temperature Materials and Precision Forming, School of Materials Science and Engineering, Shanghai Jiao Tong University, Shanghai 200240, P. R. China.

${ }^{*}$ Corresponding author. E-mail: jsun@situ.edu.cn

\section{Abstract}

The evolution of the $\omega$ phase and its influence on tensile properties in $\beta$ Ti-12V-2Fe-1Al alloys aged at temperature from 373 to $573 \mathrm{~K}$ were investigated. The results show that the formation of the thermal $\omega$ phase starts to take place at temperature between 393 and $423 \mathrm{~K}$ in the alloy. The growth of the thermal $\omega$ particles is accompanied by a rejection of $\mathrm{V}, \mathrm{Fe}$ and $\mathrm{Al}$ solute atoms from the growing thermal $\omega$ particles into the surrounding $\beta$ matrix. Tensile properties of the $\beta$ Ti-12V-2Fe-Al alloy are strongly dependent on aging temperature. The plastic deformation mode changes from fully $\{332\}$ deformation twinning in the ST alloy, to dislocation slip mixed with partially $\{332\}$ twinning in the alloy aged at $393 \mathrm{~K}$ and to dislocation slip associated with stress-induced $\omega$ phase transformation in the alloy aged at $423 \mathrm{~K}$. Particularly, the alloys aged at $523 \mathrm{~K}$ and above exhibit a brittle fracture without any elongation. It is suggested that the occurrence of the coherent elastic strain between the $\omega$ and $\beta$ phase results in stabilization effect on the $\beta$ matrix, which may account for the drastic change in plastic deformation mode and tensile properties in aged Ti- $12 \mathrm{~V}-2 \mathrm{Fe}-\mathrm{Al}$ alloys with an increase of aging temperature.

\section{Introduction}

$\beta$ Ti-based alloys are increasingly used for industrial applications, due to their low density, high strength and good corrosion resistance [1]. In general, most $\beta$ Ti-based alloys are strengthened by precipitation of the $\alpha$ phase at relatively high temperature below the $\beta$ transus temperature through thermo-mechanical treatments. However, the ductility of these alloys is limited, especially with regard to the uniform elongation. Recently, a concept in strength-ductility tradeoff was proposed for metastable $\beta$ Ti-based alloys by combining with the phase $\left(\alpha^{\prime \prime}\right)$ transformation induced plasticity (TRIP) and $\{332\}<113>$ type twinning induced plasticity (TWIP) effects through altering the stability of the $\beta$ phase with solute concentrations [2-6].

The precipitation of the $\omega$ phase with hexagonal lattice often takes place in metastable $\beta$ Ti-based alloys during quenching from the $\beta$ phase field at high temperature and during isothermal aging, which plays a significant role in their mechanical properties. It was claimed early that the formation of the thermal $\omega$ phase introduces precipitation hardening with a reduction of ductility, and even causes embrittlement after long-time aging in metastable $\beta$ Ti-11Mo and Ti-14Mo at.\% alloys aged at high temperature of 623-773 K [7]. Similar results were recently reported in the Ti-15Mo alloy aged at 523$623 \mathrm{~K}$ (all compositions are given in wt.\% except for those in at.\% hereafter) [8]. The precipitation strengthening strongly depends on the size and volume fraction of the thermal $\omega$ precipitates in those alloys, of which the mechanism was explained by the observed fact that the moving dislocations shear $\omega$ precipitates leading to an extreme inhomogeneous $\omega$-free slip distribution and cracks initiate at slip band intersections with each other and grain boundaries [7]. Such an interaction between dislocations and $\omega$ precipitates were also observed recently in the aged $\beta$ Ti-10V-2Fe-3Al alloy, where the disappearance of $\omega$ precipitates was assumed to result from the interfacial energy and dislocation slip induced $\omega$ precipitate dissolution [9], and in the $\beta$ Ti-23Nb-0.7Ta-2Zr-1.5O at.\% alloy annealed at the $\beta$ phase field and followed by furnace cooling, in which the dissociation and motion of $1 / 2[111]_{\beta}$ unit dislocations were considered to render the encountered $\omega$ precipitates transforming into the $\beta$ phase [10]. Very recently, a study of the effect of lowtemperature aging on plastic deformation in the Ti-12Mo alloy with a relatively low stability of the $\beta$ phase has been reported by Sun et al. [11]. It is shown that the stress-induced phase $\left(\alpha^{\prime \prime}\right)$ transformation is suppressed and the propensity of $\{332\}<113>$ deformation twinning decreases with 
increasing aging temperature to $523 \mathrm{~K}$. It was speculated that the elastic strain resulting from a high coherency of the $\omega$ particles embedded in the $\beta$ matrix increases rapidly during aging, prior to any detectable composition partitioning by APT, and the elastic strain energy plays a significant role in mechanical properties of the Ti-12Mo alloy [11].

In this paper, $\beta$ Ti-based alloys with a normal chemical composition of Ti-12V-2Fe-1 Al were prepared and further subjected to aging treatment at temperatures from 373 to $573 \mathrm{~K}$. The evolution of the $\omega$ phase in the alloys after aging treatments was analyzed by synchrotron X-ray diffraction (XRD), transmission electron microscopy (TEM) and atom probe tomography (APT). The influence of aging temperature on mechanical properties of the alloys was studied by tensile tests. The deformed microstructure of the alloys after tensile tests was examined by electron backscattering diffraction (EBSD) and TEM. In this paper, emphases were placed on the role of the thermal $\omega$ phase evolution in mechanical properties and plastic deformation mechanism of the alloys subjected to aging treatments.

\section{Experimental}

$\beta$ Ti-based alloys with a normal chemical composition of Ti-12V-2Fe-1 Al (wt.\%) with molybdenum equivalency (MoE) of 12.84 were prepared by using cold crucible levitation melting method under an $\mathrm{Ar}$ atmosphere from high purity $\mathrm{Ti}, \mathrm{V}, \mathrm{Fe}$ and $\mathrm{Al}$ raw metals. The alloy ingots were hot-rolled into plates at about $1223 \mathrm{~K}$ followed by air cooling. Subsequently, the alloy plates were cold-rolled with a total strain of about $30 \%$ at room temperature, and then solution-treated at $1073 \mathrm{~K}$ for 5 minutes followed by water-quenching. The solution-treated (ST) alloys were further subjected to aging treatments at temperature from 373 to $573 \mathrm{~K}$ for 1 hour, respectively. Plate-like tensile specimens with a gauge dimension of $20 \times 4 \times 2 \mathrm{~mm}^{3}$ were cut by electric discharge machine. Tensile tests were performed on Zwick/Roell Z020 machine with extensometer under a strain rate of $5 \times 10^{-4} \mathrm{~s}^{-1}$ at room temperature. Optical metallography $(\mathrm{OM})$ observations were performed using Kroll reagent $\left(5 \mathrm{HF}: 10 \mathrm{HNO}_{3}: 85 \mathrm{H}_{2} \mathrm{O}\right)$. Synchrotron XRD experiments were carried out in transmission mode at the beam line BL14B1 in the Shanghai Synchrotron Radiation Facility. The energy of the monochromatic Xray beam is about $18 \mathrm{KeV}$, corresponding to a wavelength of $0.689 \AA$. The diffraction patterns of each sample were recorded with an exposure time of 3 $5 \mathrm{~s}$. The calibration was done by using the $\mathrm{LaB} 6(\mathrm{a}=4.157 \AA)$ powder standard sample. EBSD samples were mechanically ground using 4000 grid $\mathrm{SiC}$ emery papers and then electro-polished in a methanol solution of $6 \%$ perchloric acid, $34 \% \mathrm{n}$-butyl alcohol and $60 \%$ methyl alcohol (in volume) at $-35^{\circ} \mathrm{C}$ and $20 \mathrm{~V}$. The tensile direction of tensile specimens was set to be parallel to the rolling direction (RD) for EBSD measurements. The EBSD maps were taken by using FEI Nova Nano SEM 230 scanning electron microscope (SEM) equipped with HKL Channel 5. TEM foils were first mechanically thinned to about $60 \mu \mathrm{m}$ and then finally thinned using twin-jet electro-polisher in the same electrolyte solution at $-30^{\circ} \mathrm{C}$ and $30 \mathrm{~V}$. TEM observations were performed on JEOL2100F TEM machine operating at $200 \mathrm{kV}$. Atom probe tomography (APT) needle tips with diameter less than $100 \mathrm{~nm}$ were extracted from the ST sample and samples aged at 423 and $573 \mathrm{~K}$, respectively via a dual-beam focused-ion beam (FIB) using FEI Helios Nano-Lab $600 \mathrm{i}$ device. APT experiments were performed by using LEAP 3000X HR device in the laser-pulsing mode at $250 \mathrm{KHz}$ pulse rate with 50 PJ pulse energy. Data reconstruction and analyses were conducted with IVASTM 3.6.2 software.

\section{$\underline{\text { 3. Results and discussion }}$}

The initial microstructure of the ST Ti-12V-2Fe-1 Al alloy has a fully recrystallized and equiaxed $\beta$ grain microstructure. Dark-field TEM micrographs of nanometer-sized $\omega$ particles in the ST alloy and alloys aged at 393, 423 and 573K, respectively are shown in Figs. 1(a-d). The differences of the size of the $\omega$ particles among the ST and aged samples at 393 and $423 \mathrm{~K}$ cannot be distinguished, but the size of $\omega$ particles increases markedly in the sample aged at $573 \mathrm{~K}$. The density of the $\omega$ particles in the aged sample increases very slightly with an increase of aging temperature from 393 to $573 \mathrm{~K}$. The lattice parameters of the $\beta$ and $\omega$ phase of the ST and aged alloys were determined from synchrotron XRD spectra, as shown in Table 1. It can be seen that the variation of lattice parameters of the $\beta$ phase among the ST sample and samples aged at $393 \mathrm{~K}$ and below is ignorable. With an increase of aging temperature from 423 to $573 \mathrm{~K}$, the lattice parameters of the $\beta$ phase decrease slightly. TEM and synchrotron XRD results indicate that the formation of the thermal $\omega$ phase starts to occur at temperature between 393 and $423 \mathrm{~K}$. No significant variation trend was found for the lattice parameters of the $\omega$ phase in the ST and aged samples. 

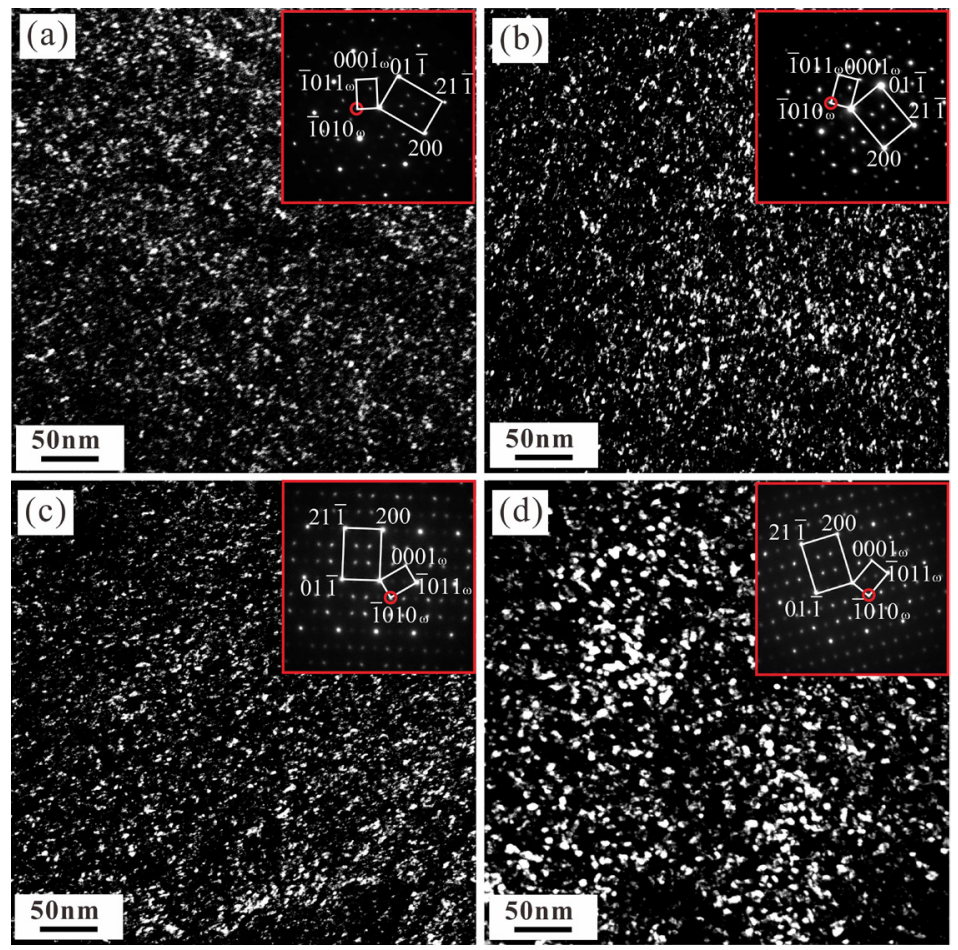

Fig. 1 Dark-field TEM micrographs of the $\omega$ phase recorded from $(-1010)_{\omega}$ reflections with corresponding $[011]_{\beta} / /[-12-10]_{\omega}$ SAED patterns as insets in the ST alloy (a) and alloys aged at $393 \mathrm{~K}(\mathrm{~b}), 423 \mathrm{~K}$ (c) and $573 \mathrm{~K}$ (d), respectively.

Table 1 Lattice parameters of the $\omega$ and $\beta$ phase in the ST alloy and alloys aged at different temperatures determined by synchrotron XRD.

\begin{tabular}{ccccc}
\hline \multirow{2}{*}{ Alloys } & \multicolumn{4}{c}{ Lattice parameter $(\AA)$} \\
\cline { 2 - 5 } & $a_{\beta}$ & $a_{\omega}$ & $c_{\omega}$ & $c_{\omega} / a_{\omega}$ \\
\hline ST & 3.228 & 4.572 & 2.811 & 0.615 \\
$373 \mathrm{~K}$ & 3.226 & 4.629 & 2.808 & 0.607 \\
$393 \mathrm{~K}$ & 3.228 & 4.587 & 2.809 & 0.612 \\
$423 \mathrm{~K}$ & 3.223 & 4.519 & 2.808 & 0.621 \\
$473 \mathrm{~K}$ & 3.221 & 4.500 & 2.803 & 0.623 \\
$523 \mathrm{~K}$ & 3.216 & 4.559 & 2.799 & 0.614 \\
$573 \mathrm{~K}$ & 3.214 & 4.607 & 2.799 & 0.608 \\
\hline
\end{tabular}

Figs. 2(a-c) show APT results of the ST alloy and alloys aged at 423 and 573K, respectively. Binomial analyses, measuring the composition by dividing the entire 3D volume into volume elements (or voxels) containing 100 atoms in this case were carried out to examine the solute distribution, and the vanadium concentration in each block is plotted as a frequency distribution. It is seen that the experimentally observed distribution of vanadium 
is identical to the calculated one for a perfectly random solid solution (standard binomial distribution), implying that the chemical composition is statistically homogenous in the ST alloy and the alloy aged at $423 \mathrm{~K}$. In contrast, the observed distribution of vanadium derives from the standard binomial distribution, indicating that there is an obvious composition partitioning of $\mathrm{V}, \mathrm{Fe}$ and $\mathrm{Al}$ elements between the solute-depleted $\omega$ and the soluterich $\beta$ phase, and the growth of the thermal $\omega$ particles is accompanied by a rejection of $\mathrm{V}, \mathrm{Fe}$ and $\mathrm{Al}$ solute atoms from the growing thermal $\omega$ particles into the surrounding $\beta$ matrix in the alloy aged at $573 \mathrm{~K}$.
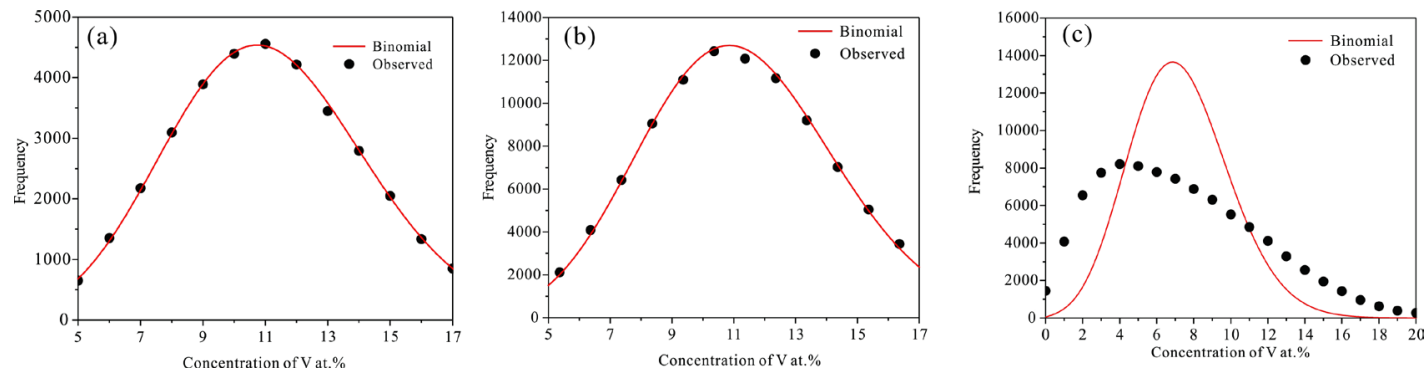

Fig. 2 Binomial distribution of the V concentration in the ST alloy (a) and alloys aged at $423 \mathrm{~K}$ (b) and $573 \mathrm{~K}$ (d), respectively.

Tensile stress-strain curves of the ST and aged Ti-12V-2Fe-1Al alloys at different aging temperatures are plotted in Fig. 3. The alloys display continuously yielding and work hardening followed by work softening, except for the alloy aged at $473 \mathrm{~K}$, which shows a upper yielding on the stressstrain curve. Particularly, the alloys aged at 523 and $573 \mathrm{~K}$ exhibt a brittle fracture without any elongation, respectively. The work hardening rate of the ST alloy is higher than those of the aged alloys. The work hardening rate of the aged alloys decreases with an increase of aging temperature. The yield strength of the ST alloy is lower, but the elongation is higher than those of the aged alloys. The yield strength of the aged alloys increase, while the tensile elongation decreases obviously with an increase of aging temperature.

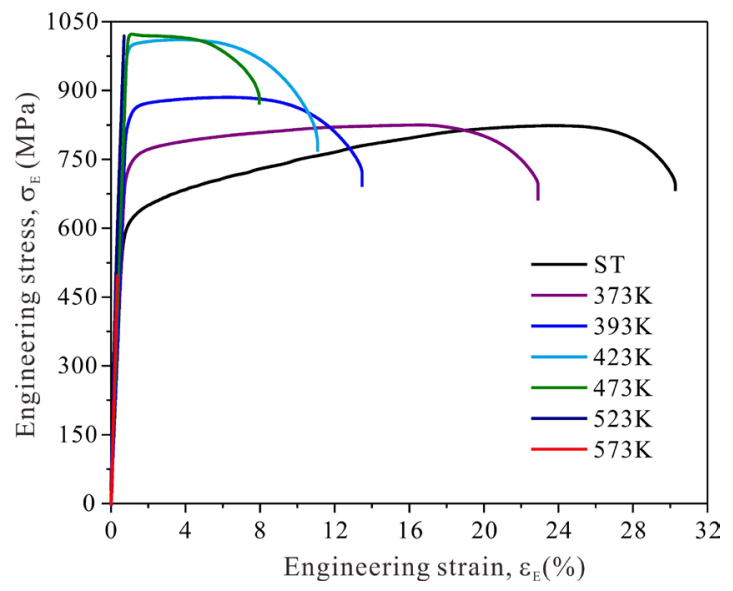

Fig. 3 Engineering stress-strain curves of the ST alloy and alloys aged at different temperatures.

Figs. 4(a-c) show EBSD image quality maps in the deformed ST sample and samples aged at 393 and $423 \mathrm{~K}$, respectively. The plate-like products within $\beta$ grains in the ST sample and aged sample at $393 \mathrm{~K}$ were identified as $\{332\}<113>$ deformation twins by EBSD analyses. The density of the $\{332\}<113>$ deformation twins are relatively lower in the sample aged at $393 \mathrm{~K}$ than that in the ST sample, and the width of the twins is wider in the 
aged sample than that in the ST sample. In the sample aged at $423 \mathrm{~K}$ after tensile tests, very thin plate-like stress-induced features appear within $\beta$ grains, however, these plate-like features are too narrow to be identified by EBSD measurements. Figs. 5(a-b) show dark-field TEM micrographs of thin platelike stress-induced products and the corresponding SAED pattern as an inset in the sample aged at $423 \mathrm{~K}$ after tensile tests. Very thin plate-like features with a width of about several nanometers on (112) planes are visible. In contrast to that two variants of the $\omega$ phase were observed in the surrounding $\beta$ matrix in Figs. 5(a, b), only one variant of the $\omega_{2}$ phase can be found for the thin plate-like features in the sample aged at $423 \mathrm{~K}$ in Fig. 5(b). This means that thin plate-like stress-induced $\omega_{2}$ phase transformation takes place on the $(112)_{\beta}$ plane in the $\beta$ matrix. The $(112)_{\beta}$ slip plane and [111 $]_{\beta}$ slip direction of the $\beta$ matrix correspond to $(1010) \omega_{2}$ plane and $[0001] \omega_{2}$ direction of the $\omega$ phase, respectively, i.e., $\left.(112)\right)_{\beta} / /(10-10) \omega_{2}$ and $[111]_{\beta} / /[0001] \omega_{2}$. It has been proposed that the stress-induced omega phases can be formed by a dislocation mechanism, i.e., a three-layer inhomogeneous shear of 1/12[111], $1 / 3[111]$, and $1 / 12$ [111] on consecutive $\{112\}$ planes in the bcc structured metals $[12,13]$. This mechanism can explain the occurrence of the thin platelike stress-induced $\omega$ phase in the alloy aged at $423 \mathrm{~K}$ under tensile deformation.
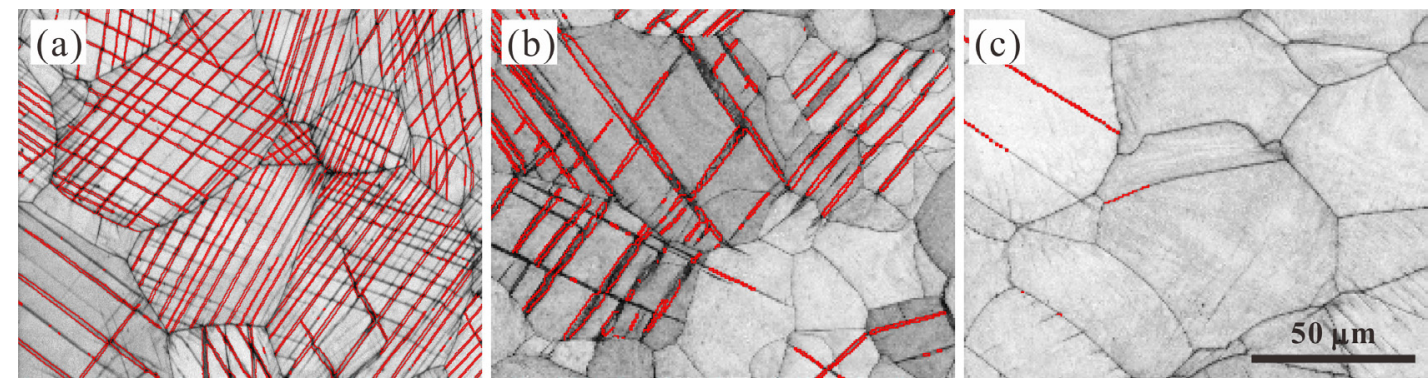

Fig. 4 EBSD image quality maps overlaid with $\Sigma 11$ boundaries (marked with red lines) in the ST alloy (a) and alloys aged at $393 \mathrm{~K}$ (b) and $423 \mathrm{~K}$ (d), respectively.
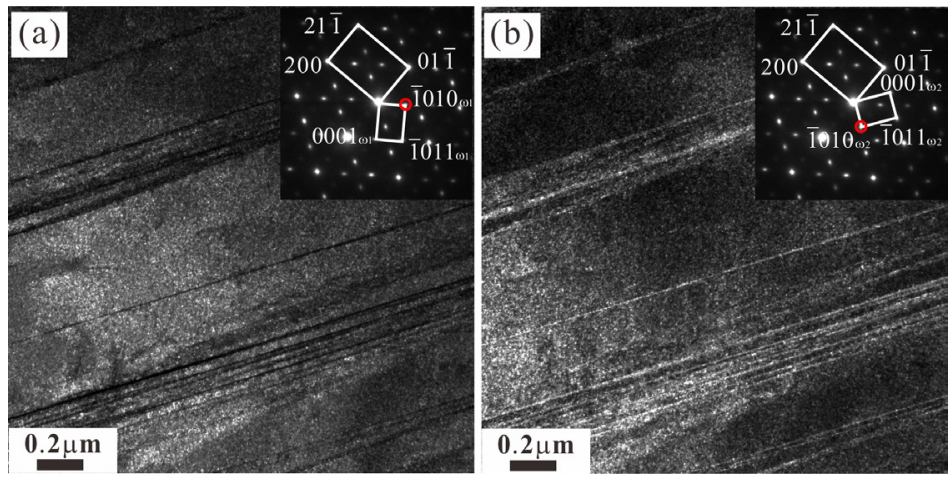

Fig. 5 Dark-field TEM micrographs recorded from $(-1010)_{\omega 1}$ (a) and $(-1010)_{\omega 2}$ reflections $(b)$ in the alloy aged at $423 \mathrm{~K}$ after tensile tests.

The results of this work show that tensile properties of the $\beta$ Ti-12V-2Fe-Al alloy are strongly dependent on aging temperature. Correspondingly, the plastic deformation mode changes from fully $\{332\}$ deformation twinning in the ST alloy, to dislocation slip mixed with partially $\{332\}$ twinning in the alloy aged at $393 \mathrm{~K}$ and to dislocation slip associated with stress-induced $\omega$ phase transformation in the alloy at $423 \mathrm{~K}$. It has been previously reported that the plastic deformation mode changes from stress-induced $\omega$ phase transformation and $\{332\}<113>$ type deformation twinning to dislocation slip with an increase of the concentration of vanadium, i.e., of the stability of the $\beta$ phase in metastable $\beta$ Ti-V alloys [14-16]. The plastic deformation of the $\beta$ phase significant depends on its phase stability, which governs mechanical properties of the metastable $\beta$ Ti-V alloys. However, 
APT measurements exhibited that there is minimal, if any, composition partitioning between the $\omega$ and $\beta$ phase in the alloy aged at $423 \mathrm{~K}$, as mentioned above. Thus, the drastic variations in tensile properties and plastic deformation mechanism cannot be explained only by the change of the $\beta$ phase stability resulting from the composition partitioning in the aged Ti-12V-2Fe-Al alloys.

It is well recognized that the composition partitioning between the solute-depleted $\omega$ and solute-rich $\beta$ phase in the alloy during aging process will result in a difference in lattice parameter between these two phases. Because the solute-depleted $\omega$ phase remains fully coherent with the solute-rich $\beta$ matrix during the nucleation and growth, there will be a strain energy associated with the formation of the thermal $\omega$ phase, and the coherent strain between these two phases will also introduce a hydrostatic pressure on the $\omega$ particles [17-18]. Certainly, the coherent strain between the $\omega$ and $\beta$ phase increases markedly with increasing aging temperature. Since the strain energy associated with the formation of the thermal $\omega$ phase and the hydrostatic pressure on the thermal $\omega$ particles in the aged alloy are several orders of magnitude larger than those for the athermal $\omega$ phase in the ST alloy [17-18], the drastic changes in tensile properties and plastic deformation mechanism may be related to the coherent strain between the $\omega$ and $\beta$ phase in the aged Ti-12V-2Fe-Al alloys in comparison with the ST alloy. On the other hand, the composition of the thermal $\omega$ phase changes significantly in the alloy aged at high temperature [17-18] and the evolution of the $\omega$ phase will change mechanical properties of $\omega$ precipitates themselves during aging process. The compositional dependence of elastic properties of the $\omega$ phase in the Ti-V alloys was previously calculated by using the first-principles theory, showing that the mechanical stability of the $\omega$ phase decreases with decreasing the concentration of vanadium [19]. Meanwhile, the shear and Young's modulus decrease, but the bulk modulus increases with increasing the concentration of vanadium [19], as shown in Figs. 6. Particularly, the G/B ratio decreases with increasing the concentration of vanadium. Pugh proposed a criterion for the ductile-brittle transition of materials by means of the G/B value, that is, a material is brittle when its G/B ratio is greater than 0.5 , otherwise it is ductile [20]. Thus, according to the Pugh's criterion, the athermal $\omega$ phase is deformable or ductile in the ST alloy, while the thermal $\omega$ phase may be undeformable or brittle due to the composition partitioning between the solutedepleted $\omega$ and solute-rich $\beta$ phase in the alloy aged at high temperature of $573 \mathrm{~K}$. It can be assumed that the deformability of the $\omega$ phase decreases as the stability of the $\omega$ phase increases in the aged alloys with an increase of aging temperature. When the stability of the thermal $\omega$ phase is relatively low, the thermal $\omega$ phase is deformable and it can be cut through by moving dislocations on the slip planes, which results in $\omega$-free channels in the $\beta$ matrix of the alloys as reported in the literature [7, 9-10]. However, when the thermal $\omega$ phase is undeformable, the dislocation acts with thermal $\omega$ precipitates in the $\beta$ matrix by a by-pass mechanism in the alloy, which requiring a very high stress, according to the Orowan equation. Thus, thermal $\omega$ precipitates are effective obstacles to dislocation motion, resulting in dislocation piling-up against $\omega$ precipitates and fracturing in a brittle mode for the omega-enriched $\beta$ Ti-12V-2Fe-Al alloy aged at high temperature.

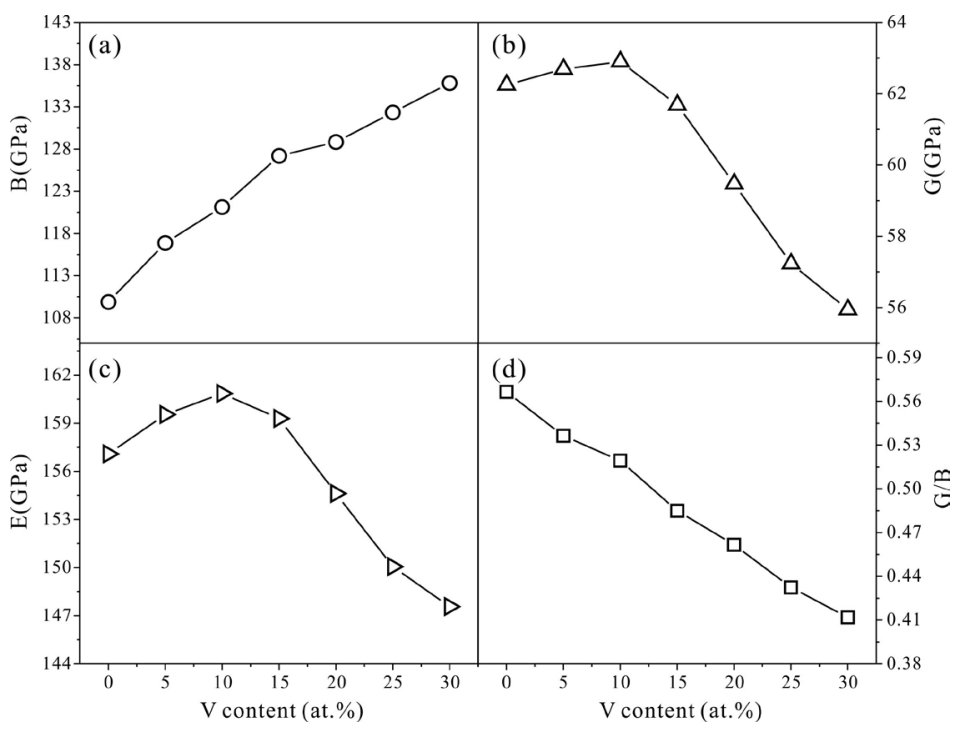


Fig. 6 The elastic modulus for the $\omega$ and $\beta$ phase in Ti-V alloys as functions of $V$ concentration: bulk modulus $B$, shear modulus $G$, Young modulus $E$ and $G / B$ ratio.

\section{Conclusions}

The formation of the thermal $\omega$ phase starts to take place at temperature between 393 and $423 \mathrm{~K}$ in the $\beta$ Ti- $12 \mathrm{~V}-2 \mathrm{Fe}-\mathrm{Al}$ alloy. The growth of the thermal $\omega$ particles is accompanied by a rejection of $\mathrm{V}, \mathrm{Fe}$ and $\mathrm{Al}$ solute atoms from the growing thermal $\omega$ particles into the surrounding $\beta$ matrix in the alloy. Tensile properties of the $\beta$ Ti-12V-2Fe-Al alloy are strongly dependent on aging temperature. The plastic deformation mode changes from fully $\{332\}$ deformation twinning in the ST alloy, to dislocation slip mixed with partially $\{332\}$ twinning in the alloy aged at $393 \mathrm{~K}$ and to dislocation slip associated with stress-induced $\omega$ phase transformation in the alloy aged at $423 \mathrm{~K}$. Particularly, the alloys aged at $523 \mathrm{~K}$ and above exhibit a brittle fracture without any elongation. It is suggested that the occurrence of the coherent elastic strain between the $\omega$ and $\beta$ phase results in stabilization effect on the $\beta$ matrix, which may account for the drastic change in plastic deformation mode and tensile properties in aged $\mathrm{Ti}-12 \mathrm{~V}-2 \mathrm{Fe}-\mathrm{Al}$ alloys with an increase of aging temperature.

\section{Acknowledgements}

The authors would like to acknowledge the financial support from the National Natural Science Foundation of China (Grant no. 51371113). The authors also would like to thank the beam line BL14B1 of Shanghai Synchrotron Radiation Facility for providing the beam time.

\section{References}

[1] C. Leyens, M. Peters, Wiley-VCH, 2003.

[2] M. Marteleur, F. Sun, T. Gloriant, P. Vermaut, P.J. Jacques, F. Prima, Scripta Mater. 66(10) (2012) 749-752.

[3] X. Min, X. Chen, S. Emura, K. Tsuchiya, Scripta Mater. 69(5) (2013) 393-396.

[4] W.L. Wang, X.B. Zhang, J. Sun, Mater Charact. 142 (2018) 398-405.

[5] W.L. Wang, X.L. Wang, W. Mei, J. Sun, Mater Charact. 120 (2016) 263-267.

[6] X.L. Wang, L. Li, H. Xing, P. Ou, J. Sun, Scripta Mater. 96 (2015) 37-40.

[7] A. Gysler, G. Lütjering, V. Gerold, Acta Metall. 22(7) (1974) 901-909.

[8] F. Brunke, C. Siemers, J. Rösler, Proceedings of the 13th World Conference on Titanium, (2015) 929-934.

[9] W. Chen, J. Zhang, S. Cao, Y. Pan, M. Huang, Q. Hu, Q. Sun, L. Xiao, J. Sun, Acta Mater. 117 (2016) 68-80.

[10] M.J. Lai, C.C. Tasan, D. Raabe, Acta Mater. 100 (2015) 290-300.

[11] F. Sun, J.Y. Zhang, P. Vermaut, D. Choudhuri, T. Alam, S.A. Mantri, P. Svec, T. Gloriant, P.J. Jacques, R. Banerjee, F. Prima, Mater. Res. Lett. 5(8) (2017) 1-7.

[12] L.M. Hsiung, D.H. Lassila, Acta Mater. 48(20) (2000) 4851-4865.

[13] L.M. Hsiung, D.H. Lassila, Scripta Mater. 39(4-5) (1998) 603-609.

[14] S. Hanada, A. Takemura, O. Izumi, The mode of plastic deformation of $\beta$ Ti-V alloys, Trans. Japan Inst. 23(9) (1982) 507-517.

[15] S. Hanada, O. Izumi, Metall. Trans. A 17(8) (1986) 1409-1420.

[16] X.L. Wang, L. Li, W. Mei, W.L. Wang, J. Sun, Mater Charact. 107 (2015) 149-155.

[17] A. Devaraj, S. Nag, R. Srinivasan, R.E.A. Williams, S. Banerjee, R. Banerjee, H.L. Fraser, Acta Mater. 60(2) (2012) 596-609.

[18] D. Choudhuri, Y. Zheng, T. Alam, R. Shi, M. Hendrickson, S. Banerjee, Y. Wang, S.G. Srinivasan, H. Fraser, R. Banerjee, Acta Mater. 130 (2017) 215-228.

[19] W. Mei, J. Sun, Y. Wen, Prog. Nat. Sci. 27(6) (2017) 703-708.

[20] S.F. Pugh, Philos. Mag. 45(367) (1954) 823-843. 